\title{
MiRNA-210 modulates a nickel-induced cellular energy metabolism shift by repressing the iron-sulfur cluster assembly proteins ISCU1/2 in Neuro-2a cells
}

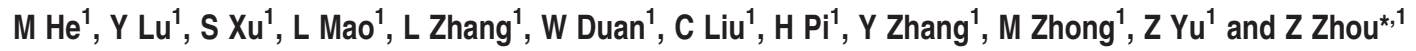

The cellular energy metabolism shift, characterized by the inhibition of oxidative phosphorylation (OXPHOS) and enhancement of glycolysis, is involved in nickel-induced neurotoxicity. MicroRNA-210 (miR-210) is regulated by hypoxia-inducible transcription factor-1 $\alpha$ (HIF-1 $\alpha$ ) under hypoxic conditions and controls mitochondrial energy metabolism by repressing the ironsulfur cluster assembly protein (ISCU1/2). ISCU1/2 facilitates the assembly of iron-sulfur clusters (ISCs), the prosthetic groups that are critical for mitochondrial oxidation-reduction reactions. This study aimed to investigate whether miR-210 modulates alterations in energy metabolism after nickel exposure through suppressing ISCU1/2 and inactivating ISCs-containing metabolic

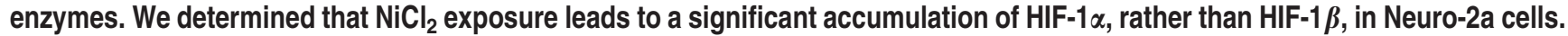
The miR-210 overexpression and ISCU1/2 downregulation was observed in a dose- and time-dependent manner. The gain-offunction and loss-of-dysfunction assays revealed that miR-210 mediated the ISCU1/2 suppression, energy metabolism alterations, and ISC-containing metabolic enzyme inactivation after nickel exposure. In addition, the impact of miR-210 on ISC-containing metabolic enzymes was independent from cellular iron regulation. Overall, these data suggest that repression of miR-210 on ISCU1/2 may contribute to HIF-1 $\alpha$-triggered alterations in energy metabolism after nickel exposure. A better understanding of how nickel impacts cellular energy metabolism may facilitate the elucidation of the mechanisms by which nickel affects the human health.

Cell Death and Disease (2014) 5, e1090; doi:10.1038/cddis.2014.60; published online 27 February 2014

Subject Category: Neuroscience

Nickel (Ni) compounds are widely distributed environmental pollutants and are hazardous to public health. ${ }^{1}$ Although the exact mechanism of Ni-induced toxicity is unknown, increasing evidence indicates that the hypoxia-mimic responses, which are primarily mediated by hypoxia-inducible factor- $1 \alpha$ $(\mathrm{HIF}-1 \alpha)$ stabilization, may contribute to the adverse effects of $\mathrm{Ni}$ on the human body. ${ }^{2,3}$ The activation of HIF-1 $\alpha$ target genes, including plasminogen activator inhibitor (PAl-1), vascular endothelial growth factor (VEGF), and the CXC chemokine, are involved in $\mathrm{Ni}$ dust-induced fibrotic lung disorders. ${ }^{4,5}$ HIF-1 $\alpha$-dependent upregulation of IL- 6 in human primary endothelial cells occurs in $\mathrm{Ni}$ contact allergic reactions. ${ }^{6}$ Moreover, HIF-1 $\alpha$-regulated angiogenesis and gene silencing may contribute to $\mathrm{Ni}$-induced carcinogenesis. ${ }^{2}$ Additional investigations of $\mathrm{Ni}$-induced hypoxia-mimic responses may elucidate the mechanisms of Ni toxicity in humans.

The alterations of energy metabolism, characterized by the repression of the tricarboxylic acid (TCA) cycle, mitochondrial electron transport, and oxidative phosphorylation (OXPHOS), but the enhancement of glycolysis, are typical adaptive responses under hypoxia-induced stress. ${ }^{7}$ Interestingly, these alterations are $\mathrm{HIF}-1 \alpha$ dependent and arise under normoxic conditions if cellular HIF- $1 \alpha$ is stabilized by chemical agents. ${ }^{8,9}$ Although the metabolic shift favors resistance and survival of cells under hypoxic conditions, ${ }^{10}$ this shift contributes to the harmful effects of $\mathrm{Ni}$. Chen and Costa ${ }^{11}$ reported that $\mathrm{Ni}$ decreases the activity of cellular ironcontaining enzymes, inhibits OXPHOS, and increases cellular glycolytic activity. The inducible adaptability of cells under hypoxic conditions facilitates their malignant transformation after chronic exposure to $\mathrm{Ni}^{2}{ }^{2}$ In addition, energy supply reduction leads to the dysfunction of tissues with high energy demand, including the nervous system. In our previous study, ingestion of soluble $\mathrm{Ni}$ compounds in mice resulted in the disturbance of aerobic metabolism accompanied by neurobehavioral changes. ${ }^{12}$ Therefore, investigation of the relationship between HIF-1 $\alpha$ stabilization and energy metabolism alteration is an ideal strategy for elucidating the mechanism of Ni toxicity.

Endogenous microRNA molecules (miRNAs) are important mediators of numerous cellular processes, including the response to hypoxia. ${ }^{13}$ MicroRNA-210 (miR-210), which is induced by HIF- $1 \alpha$ during hypoxia, is one of the most hypoxiasensitive miRNAs and is an ideal factor modulating the

\footnotetext{
${ }^{1}$ Department of Occupational Health, Third Military Medical University, Chongqing 400038, People's Republic of China

*Corresponding author: ZZhou, Department of Occupational Health, Third Military Medical University, No. 30, Gaotanyan Street, Shapingba District, Chongqing 400038, China. Tel: +86 23 68752289; Fax: +86 23 68752289; E-mail: lunazhou00@163.com

Keywords: nickel; energy metabolism shift; miR-210; ISCU1/2; glycolysis

Abbreviations: OXPHOS, oxidative phosphorylation; miR-210, microRNA-210; HIF-1 $\alpha$, hypoxia-inducible transcription factor-1 $\alpha$; ISCU1/2, iron-sulfur cluster assembly protein; ISC, iron-sulfur cluster; Ni, nickel; TCA, tricarboxylic acid; UTR, untranslated region; DFX, deferoxamine; 2-DG, 2-deoxy-D-glucose; BrPA, bromopyruvic acid; HK, hexokinase; TfR, transferrin receptor; OD, optical density; ROS, reactive oxygen species

Received 30.10.13; revised 17.1.14; accepted 20.1.14; Edited by C Munoz-Pinedo
} 
hypoxia response. ${ }^{14}$ Recent studies have investigated the repression of miR-210 on the iron-sulfur cluster (ISC) assembly proteins, ISCU1 and ISCU2, whose $3^{\prime}$-untranslated region (UTR) is predicted to contain a highly conserved binding site for miR-210. ${ }^{15,16}$ In mammalian cells, ISCU1 is located in the cytosol, whereas ISCU2 is located in the mitochondria. ISCU1/2 is a key chaperone for the assembly of cellular ISCs and transportation to enzymes that are responsible for mitochondrial respiration and energy production. ${ }^{17}$ These enzymes include aconitase, which is integral to the TCA cycle, and the mitochondrial respiratory complexes (complexes I, II, and III), which mediate mitochondrial electron transport. Therefore, the suppression of ISCU1/2 leads to the inactivation of ISC-containing enzymes by ISC metabolic disorder, and ultimately inhibits mitochondrial respiration. The regulation axis comprising HIF- $1 \alpha$, miR-210, and ISCU1/2 mediates the mitochondrial energy metabolism processes that respond to hypoxia in different cell types. ${ }^{18-21}$ Compared with hypoxia, $\mathrm{Ni}$ exposure can disturb cellular iron homeostasis that is essential for the metabolism of ISCs. ${ }^{22}$ The evaluation of whether the modification of cellular iron states is involved in Ni-mediated metabolic events is of interest.

Therefore, the present study aimed to investigate whether the repression of miRNA-210 on ISCU1/2 underlay a $\mathrm{Ni}$-induced energy metabolism shift. Ni-mediated miR-210 upregulation repressed the expression of ISCU1/2, inhibited the typical ISC enzymes, and shifted downstream mitochondrial metabolism. Our results indicate that miR-210 is essential and sufficient to modulate the cellular energy metabolism alterations induced by $\mathrm{Ni}$.

\section{Results}

Ni induces HIF-1 $\alpha$ stabilization and miR-210 expression. The expressions of HIF and miR-210 induced by $\mathrm{NiCl}_{2}$ treatments were investigated in Neuro-2a cells. Cells were exposed to different concentrations of $\mathrm{NiCl}_{2}(0,0.25,0.5$, and $1 \mathrm{mM})$ for $4 \mathrm{~h}$ or $1 \mathrm{mM}$ of $\mathrm{NiCl}_{2}$ for different time periods $(0,2$, 4 , and $8 \mathrm{~h}$ ). Western blot analysis revealed that $\mathrm{Ni}$ treatment resulted in obvious stabilization of $\mathrm{HIF}-1 \alpha$ but did not change the expression of HIF-1 $\beta$ (Figures $1 \mathrm{a}$ and $b$ ). Ni-induced overexpression of miR-210, identified by qRT-PCR analysis, was observed in dose- and time-dependent manners (Figures 1c and d). In addition, the established agents that stabilized HIF-1 $\alpha$, deferoxamine (DFX) and $\mathrm{CoCl}_{2}$, increased miR-210 expression after $4 \mathrm{~h}$ of treatment in Neuro-2a cells, as presented in Figure $1 e$. Ni-induced miR-210 expression represses ISCU1/2
protein levels. The protein and transcript levels of ISCU1/2
in cells with different $\mathrm{NiCl}_{2}$ treatments were investigated.
The protein levels of $\mathrm{ISCU}_{1 / 2}$ decreased in dose- and
time-dependent manners in cells that were exposed to $\mathrm{NiCl}_{2}$
(Figures $2 \mathrm{a}$ and b). The $\mathrm{Ni}$-induced activation of $\mathrm{HIF}-1 \alpha /$
miR210/ISCU regulation axis, which embodied as a
stabilization of $\mathrm{HIF}-1 \alpha$, an overexpression of miR210, and a
repression of ISCU, was identified in different cells
(Supplement Figures 1,2 and 3 ). The transcript levels of
ISCU1/2 were not significantly different among cells with
different $\mathrm{NiCl}_{2}$ treatments (Figures $2 \mathrm{c}$ and d). To determine Cell Death and Disease whether ISCU1/2 was regulated by miR-210, the wild-type and mutant (71-77 ACGCACA mutated to AGCGAGA) forms of the predicted target site in the $3^{\prime}$-UTR of ISCU1/2 (http:// www.targetscan.org/mmu_61/) were cloned into luciferase reporter gene vectors. The transfection of miR-210 mimic repressed the activity of luciferase that interacted with the wild-type miR-210 predicted target site. However, the miR210-mimic transfection did not alter the activity of luciferase that interacted with mutant target site (Figure 2e). These results verified that miR-210 regulates the expression of ISCU1/2 by binding to the predicted target site in the $3^{\prime}$-UTR of ISCU1/2. The analysis of protein level in cells that were transfected with the miR-210 mimic or inhibitor was performed to further establish that ISCU1/2 was the downstream target of miR-210. Under non-Ni exposure conditions, the transfection of miR-210 mimic (denoted as $\mathrm{M}$ ) suppressed the ISCU1/2 protein level compared with that of the mimic-control (MC). Furthermore, under $\mathrm{Ni}$ exposure conditions (1 mM for $4 \mathrm{~h}$ ), the transfection of the miR-210 inhibitor (I) attenuated the $\mathrm{Ni}$-induced ISCU1/2 downregulation compared with that of the inhibitor-control (IC, Figure 2f).

Ni-induced miR-210 expression modulates the shift of energy metabolism. The $\mathrm{Ni}$-induced shift of energy metabolism, characterized as the inhibition of OXPHOS and increase in glycolysis, plays important roles in Ni-mediated neurotoxicity and carcinogenesis. In our study, the viability of Neuro-2a cells that were treated with $1 \mathrm{mM} \mathrm{NiCl}_{2}, 10 \mathrm{mM}$ 2-deoxy-D-glucose (2-DG), or $25 \mu \mathrm{M}$ bromopyruvic acid (BrPA) was equivalent to control levels. However, $\mathrm{Ni}$ exposure combined with 2-DG or BrPA, both of which are inhibitors of glycolysis, resulted in significant cytotoxicity in Neuro-2a cells (Figure 3a). The cells exposed to $1 \mathrm{mM} \mathrm{NiCl}_{2}$ for $4 \mathrm{~h}$ also manifested a low oxygen consumption rate (OCR) (Figure 3b). To confirm that the Ni-induced energy metabolism shift was modulated by miR-210 expression, we investigated the energy metabolism states of cells that were transfected with miR-210 mimic or inhibitor and treated with $\mathrm{NiCl}_{2}$. The quantifications of OCR revealed that oxygen consumption was inhibited in cells that were transfected with miR-210 mimic or treated with $\mathrm{NiCl}_{2}$. The transfection of miR-210 inhibitor eased off the suppression of $\mathrm{Ni}$ on oxygen consumption (Figure 3c). The transfection of miR-210 mimic reduced the concentration of cellular adenosine-5'-triphosphate (ATP) under non-Ni conditions, whereas inhibition of miR-210 attenuated the ATP depletion caused by $\mathrm{Ni}$ (Figure $3 d$ ). Hexokinase (HK) is the key enzyme in glycolysis, and an increase in HK activity indicates the enhancement of glycolysis. The transfection of miR-210 mimic enhanced the activity of $\mathrm{HK}$ under non- $\mathrm{NiCl}_{2}$ conditions, whereas inhibition of miR-210 mitigated the HK activity increase caused by $\mathrm{Ni}$ (Figure $3 e$ ). The transfection of miR-210 mimic increased the content of cellular lactic acid, the end product of glycolysis, whereas the inhibition of miR-210 attenuated the lactic acid accumulation caused by Ni (Figure 3f).

Ni-induced miR-210 expression inhibits the ISCcontaining metabolic enzymes. The activity of two typical ISC-dependent metabolic enzymes, aconitase and complex I, in cells transfected with miR-210 mimic and inhibitor was 


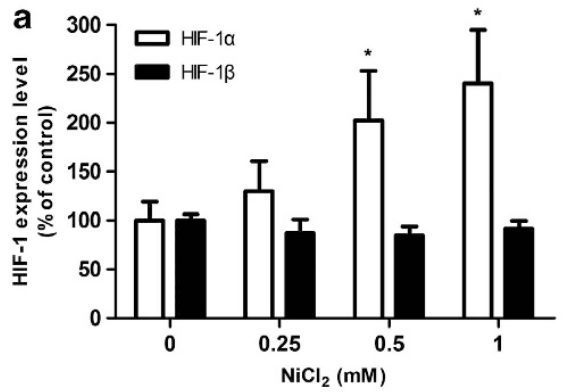

b
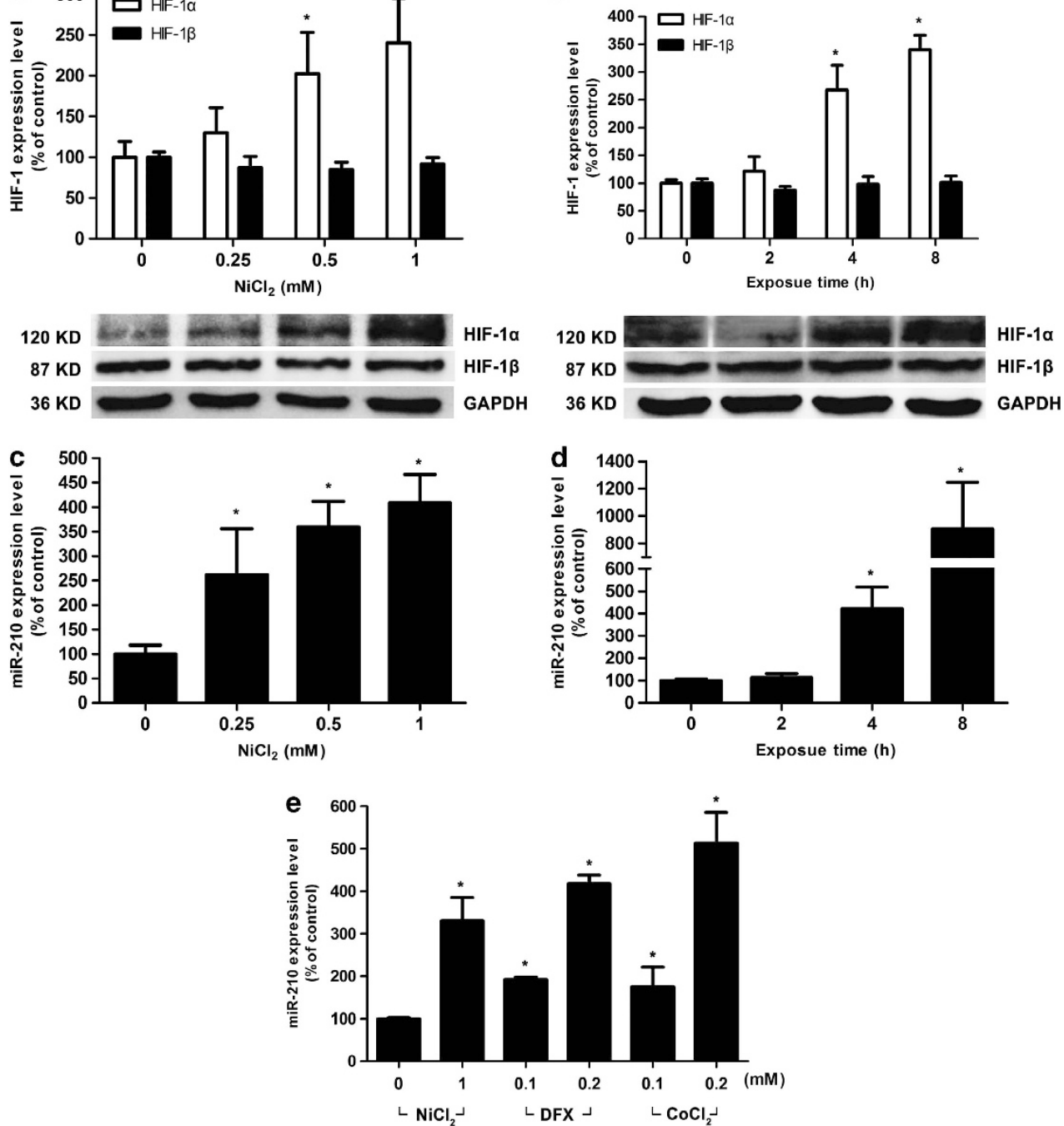

Figure 1 Nickel induces HIF-1 $\alpha$ stabilization and miR-210 expression. ( $\mathbf{a}$ and $\mathbf{b}$ ) The HIF-1 $\alpha$ and HIF-1 $\beta$ protein levels were detected by western blot after different NiCl 2 treatments. The nickel-induced HIF-1 $\alpha$ stabilizations are indicated by quantification of western blot band intensity and typical images. (c and d) The miR-210 expression after different $\mathrm{NiCl}_{2}$ treatments was analyzed by qRT-PCR. The nickel-induced upregulation of miR-210 is both dose and time dependent. (e) The well-established agents that stabilized cellular HIF-1 $\alpha$, DFX and $\mathrm{CoCl}_{2}$, also increased miR-210 expression after $4 \mathrm{~h}$ of treatment. The error bar reflects the S.E.M. of at least three independent experiments. ${ }^{*} P<0.05$ compared with the control

measured. The transfection of miR-210 mimic suppressed the activity of these two enzymes under non-Ni conditions, whereas inhibition of miR-210 attenuated the suppression of enzymes caused by Ni. Furthermore, the transfection or treatment did not change the protein level of the representative component of aconitase (ACO2) or complex I (NDUFA9), as depicted in Figures $4 \mathrm{c}$ and $\mathrm{d}$.

Treatment with $1 \mathrm{mM} \mathrm{NiCl}$ for $4 \mathrm{~h}$ does not disturb cellular iron homeostasis. To determine whether the $\mathrm{Ni}$-mediated ISC-containing enzyme suppression relates to the Ni-caused iron homeostasis disturbance, the iron metabolism states of cells exposed to $1 \mathrm{mM} \mathrm{NiCl} \mathrm{N}_{2}$ for different times were examined. A significant decrease of cellular iron ions is only found in the $8 \mathrm{~h}$ exposure groups (Figure 5a). The levels of total ferritin protein, the major form of stored iron in cells, were analyzed by western blot after
Ni treatment. $\mathrm{NiCl}_{2}$ exposure for 2 or $4 \mathrm{~h}$ did not reduce cellular ferritin levels. However, the level of ferritin in cells that were exposed to $\mathrm{Ni}$ for $8 \mathrm{~h}$ was reduced (Figure $5 \mathrm{~b}$ ). The overexpression of the transferrin receptor (TfR), an important cellular response under iron-deficient conditions, was only observed in cells exposed to $\mathrm{NiCl}_{2}$ for $8 \mathrm{~h}$ (Figure $5 \mathrm{c}$ ).

\section{Discussion}

In this study, treatment with $1 \mathrm{mM} \mathrm{NiCl}_{2}$ for $4 \mathrm{~h}$ induced $\mathrm{HIF}-1 \alpha$ stabilization, miR-210 overexpression, ISCU1/2 protein reduction, energy metabolism alterations, and ISC-containing metabolic enzyme suppression in Neuro-2a cells. Although the Ni levels in our study are unlikely to be achieved in vivo, such levels of $\mathrm{Ni}$ exposure are commonly applied in in vitro study to investigate the mechanism of Ni toxicity. ${ }^{23-26}$ Our previous study revealed that $1 \mathrm{mM} \mathrm{NiCl}_{2}$ exposure for more 

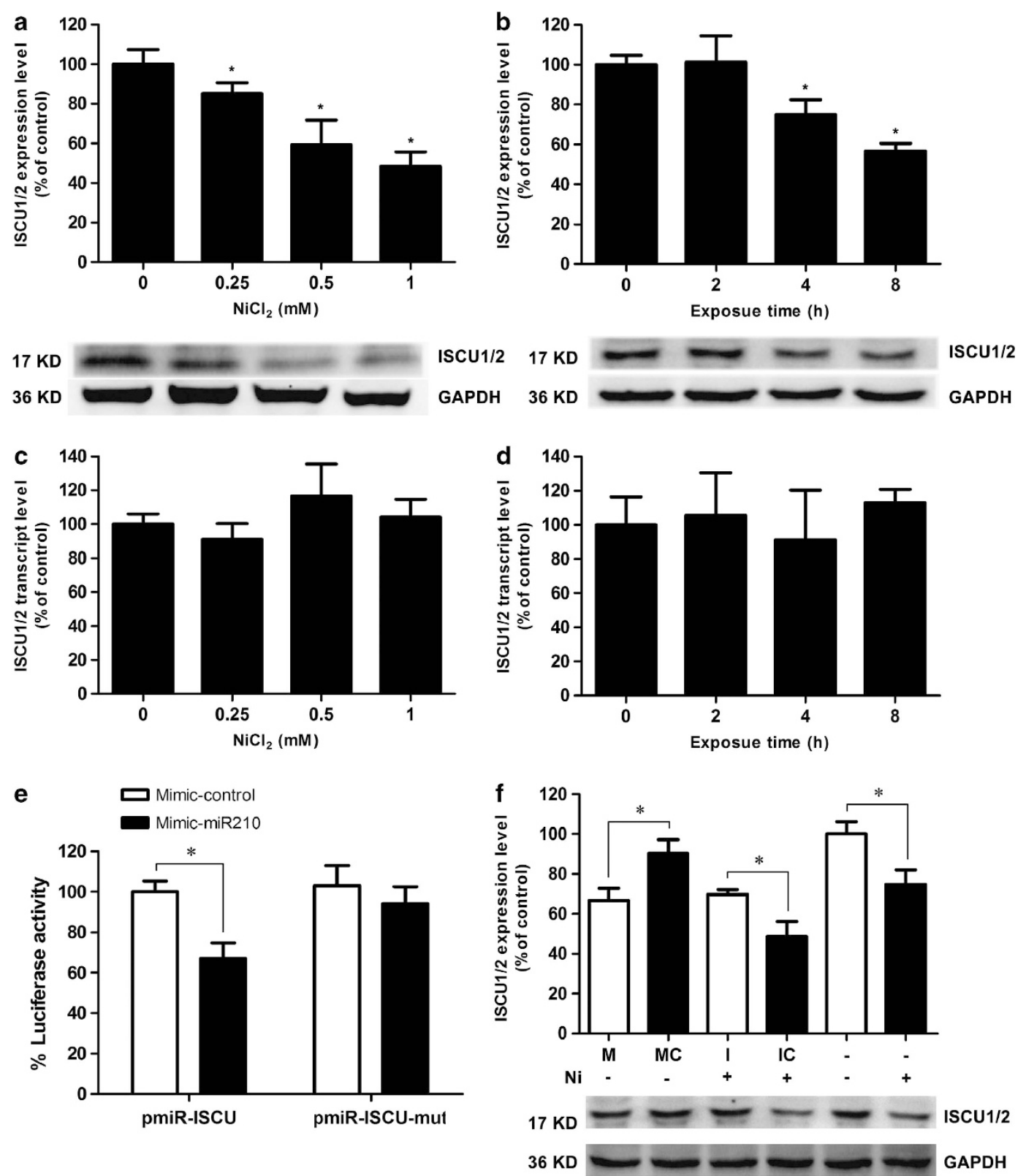

Figure 2 Nickel-induced miR-210 expression represses the level of ISCU1/2 protein. (a and $\mathbf{b}$ ) The ISCU1/2 protein levels decreased after different $\mathrm{NiCl}_{2}$ treatments. (c and d) The transcript levels of ISCU1/2 do not change under any nickel exposure conditions. (e) The effect of miR-210 on ISCU1/2 was assessed using the luciferase reporter assay. The decrease of luciferase activity in cells that were co-transfected with the wild-type ISCU1/2 $3^{\prime}$-UTR containing constructs and miR-210 mimic indicated that ISCU1/2 was a target gene of miR-210. (f) Western blotting revealed the effects of the miR-210 mimic or inhibitor transfection on the ISCU1/2 protein levels. ISCU1/2 is repressed by miR-210 mimic compared with the mimic-control (the left two lanes). During nickel exposure, the inhibition of miR-210 better preserves the level of ISCU1/2 compared with the inhibitor-control (the middle two lanes). The effect of nickel on the ISCU1/2 level was also evaluated in nontransfected cells (the right two lanes). The abbreviations M, MC, I, and IC indicate the transfection of miR-210 mimic, mimic-control, miR-210 inhibitor, and inhibitor-control, respectively. The sign ' + ' indicates the treatment of $1 \mathrm{mM} \mathrm{NiCl}_{2}$ for $4 \mathrm{~h}$, and the sign ' - ' indicates the corresponding sham treatment. The error bar reflects the S.E.M. of at least three independent experiments. ${ }^{*} P<0.05$ compared with the control

than $12 \mathrm{~h}$ produced obvious cytotoxicity and mitochondrial dysfunction in Neuro-2a cells. ${ }^{27}$ Because energy metabolism plays an important role in physiological processes of the brain, investigating energy metabolism alterations before cell death occurs may contribute to the elucidation of the mechanisms of Ni-induced neurotoxicity.

Similar to hypoxia, Ni also suppresses the activity of prolyl hydroxylase and disrupts the degradation of HIF-1 $1 \alpha$. Several studies revealed that brief (2-6 h) exposures to Ni caused the accumulation of HIF-1 $\alpha$ in various types of cell and triggered the HIF-1 $\alpha$-modulating hypoxia-mimic responses. ${ }^{5,28,29}$ Therefore, the evidence of cellular responses to hypoxia is applied to explain the health effects of $\mathrm{Ni}$. The elucidation of miR-210, a HIF- $1 \alpha$-controlled microRNA, substantially clarifies the nature of the varied cellular responses to hypoxia. ${ }^{9,30}$ MiR-210 targets the receptor tyrosine kinase ligand EphrinA3 
a

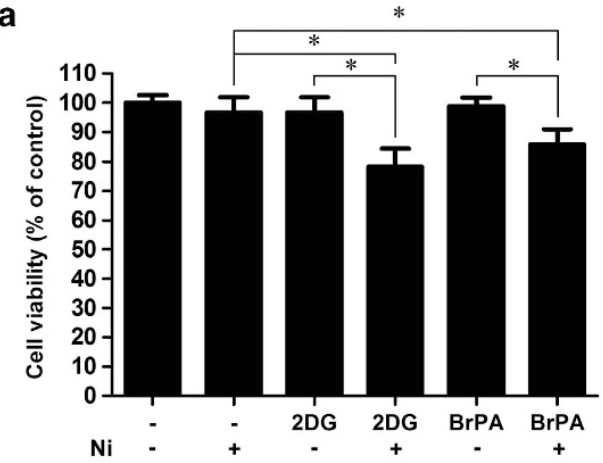

C

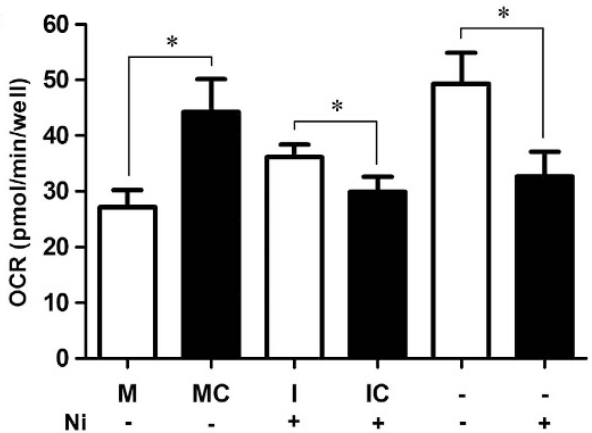

e

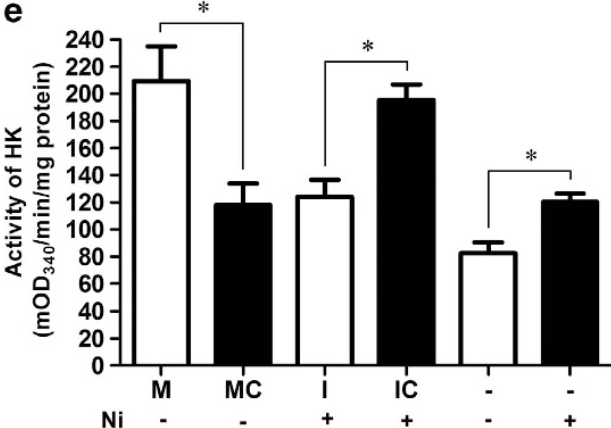

b

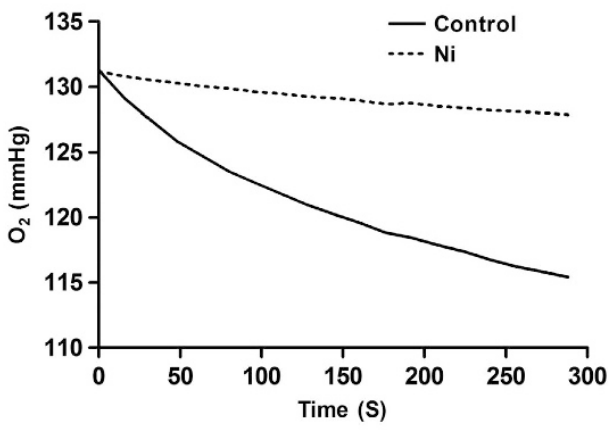

d

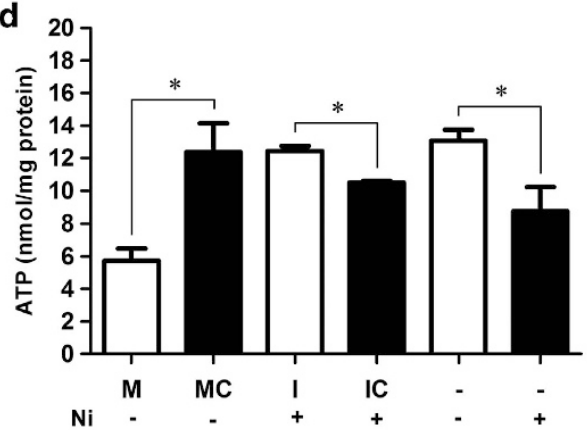

f

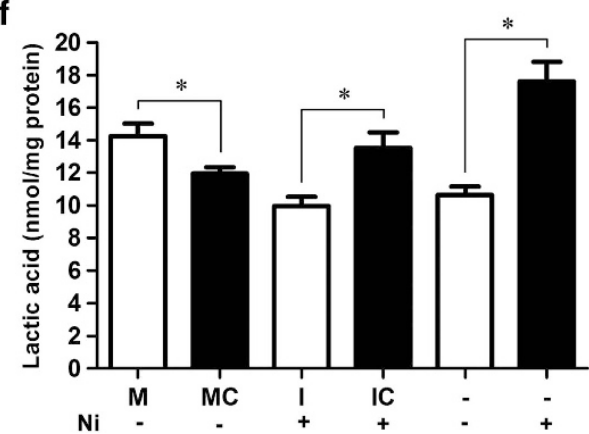

Figure 3 Nickel-induced miR-210 expression modulates the shift of energy metabolism. (a) Cell viability was determined by the CCK8 assay after nickel treatment. The inhibitors of glycolysis, 2-DG $(10 \mathrm{mM})$ or $\operatorname{BrPA}(25 \mu \mathrm{M})$, exacerbate the cytotoxicity of nickel. (b) A representative oxygen consumption curve that bases on the real-time detected partial pressure of oxygen $(\mathrm{mmHg})$ surrounding the cells indicates the suppression of nickel on cellular oxygen consumption. (c) Transfection of miR-210 mimic reduces the oxygen consumption rate (OCR) of cells in non- $\mathrm{NiCl}_{2}$ conditions, and inhibition of miR-210 eases off the repression of oxygen consumption rate caused by nickel exposure. (d) The transfection of miR-210 mimic reduces the concentration of cellular ATP in non- $\mathrm{NiCl}_{2}$ conditions, and inhibition of miR-210 attenuates the ATP depletion caused by nickel exposure. (e) The activity of hexokinase (HK) was measured based on the coupled reactions of HK and 6-phosphoglucose dehydrogenase and reflected by the change of optical density at $340 \mathrm{~nm}\left(\Delta \mathrm{mOD}_{340} / \mathrm{min} / \mathrm{mg}\right)$ measuring the generation of $\mathrm{NADPH}$. The transfection of miR-210 mimic enhances the activity of $\mathrm{HK}$ in non-NiCl 2 conditions, and inhibition of miR-210 mitigates the HK activity increase caused by nickel exposure. (f) The transfection of miR-210 mimic increases the content of cellular lactic acid in non- $\mathrm{NiCl}_{2}$ conditions, and inhibition of miR-210 attenuates the lactic acid accumulation caused by nickel exposure. The abbreviations $\mathrm{M}, \mathrm{MC}$, I, and IC indicate the transfection of miR-210 mimic, mimic-control, miR-210 inhibitor, and inhibitor-control, respectively. The sign ' + 'indicates the treatment of $1 \mathrm{mM} \mathrm{NiCl}$ for $4 \mathrm{~h}$, and the sign ' - ' indicates the corresponding sham treatment. The error bar reflects the S.E.M. of at least three independent experiments. ${ }^{*} P<0.05$ compared with the control

(EFNA3), enhancing the differentiation of human umbilical vein endothelial cells and promoting angiogenesis in hypoxia. $^{31,32}$ In addition, hypoxia-induced miR-210 participates in cell cycle regulation by modulating its target genes, MNT, a known MYC antagonist, and E2F3, a key protein in cell cycle regulation. ${ }^{33,34}$ Under oxygen/glucose deprivation conditions, the overexpressed miR-210 represses the expression of Bcl-2 and amplifies apoptosis of Neuro-2a cells. ${ }^{35}$ In our study, HIF-1 $\alpha$ stabilization and miR-210 overexpression induced by $\mathrm{NiCl}_{2}$ were observed in dose- and time-dependent manners. Moreover, $\mathrm{CoCl}_{2}$ and DFX, the well-established hypoxia-mimic compounds, increased the expression of miR-210. Therefore, miR-210 may contribute to the chemical hypoxia responses, in addition to the low oxygen content.

The energy metabolism shift is an important cellular response to hypoxia. When oxygen is abundant, cellular ATP is primarily generated through OXPHOS that generates 32 moles of ATP from 1 mole of glucose. Under hypoxic conditions, OXPHOS is suppressed and cellular energy production shifts to the glycolytic pathway that only generates 2 moles of ATP from 1 mole of glucose. Although glycolysis is not economic for ATP generation, it provides the minimum 
a

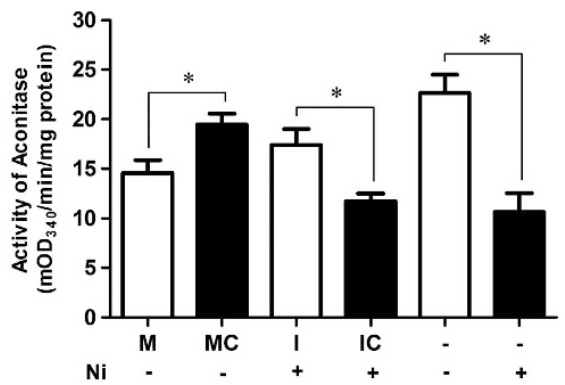

C

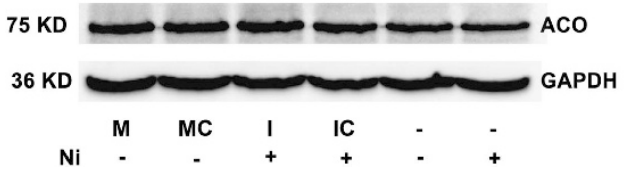

b

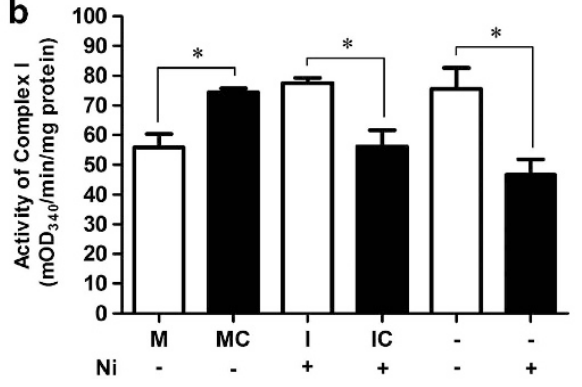

d

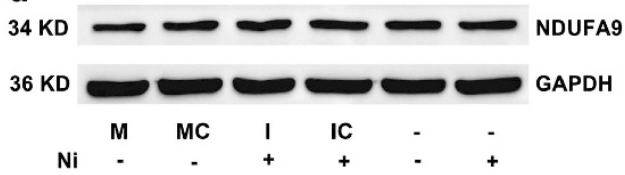

Figure 4 Nickel-induced miR-210 expression inhibits the ISC-containing metabolic enzymes. (a and b) Aconitase and complex I activity of cells transfected with miR-210 mimic or inhibitor was measured using a spectrophotometric method and expressed as $\Delta \mathrm{mOD}_{340} / \mathrm{min} / \mathrm{mg}$ of protein. (c and $\mathbf{d}$ ) The representative bands by western blot analysis are presented to demonstrate the protein levels of aconitase (ACO-2) or complex I (NDUFA9) after appropriate treatments. The abbreviations M, MC, I, and IC indicate the transfection of miR-210 mimic, mimic-control, miR-210 inhibitor, and inhibitor-control, respectively. The sign ' + ' indicates treatment with $1 \mathrm{mM}$ NiCl $\mathrm{I}_{2}$ for $4 \mathrm{~h}$, and the sign ' - ' indicates the corresponding sham treatment. The error bar reflects the S.E.M. of at least three independent experiments. ${ }^{*} P<0.05$ compared with the control

energy supplement for cell survival under hypoxic conditions. ${ }^{36}$ Recently, the energy metabolic shift regulated by the HIF-1 $\alpha$-miR-210-ISCU1/2 pathway has been revealed to participate in multiple hypoxia-related physiological and pathological processes. ${ }^{18,21}$ In our study, $\mathrm{Ni}$ exposure resulted in the downregulation of ISCU1/2 protein levels, which was connected to the changes in HIF-1 $\alpha$ and miR-210. The results of the reporter gene assay for the predicted miR-210 bonding site on ISCU1/2 and the western blot analysis of ISCU1/2 protein levels after miR-210 transfection further supported that ISCU1/2 was regulated by miR-210 in $\mathrm{Ni}$ exposure conditions. The lack of change in ISCU1/2 mRNA levels after $\mathrm{Ni}$ exposure revealed the post-transcriptional regulatory effects of miR-210 on ISCU1/2. ${ }^{15}$ Moreover, the activation of $\mathrm{HIF}-1 \alpha / \mathrm{miR}-210 / \mathrm{ISCU} 1 / 2$ regulation pathway was universal in different Ni-treated cells. The downstream effects of this regulation pathway may underlie the Ni-mediated hypoxia-mimic response.

The energy metabolism shift, characterized by glycolysis enhancement under normoxic conditions, is considered to be involved in the toxicity of $\mathrm{Ni}$. In the central nervous system, $\mathrm{Ni}$-mediated inhibition of OXPHOS resulted in energy supplement stress. ${ }^{12}$ Inhibition of glycolysis with 2-DG or BrPA increased the cytotoxicity of $\mathrm{NiCl}_{2}$ in Neuro-2a cells in the present study. In addition, the cellular phenotype of preferential glycolytic energy generation, known as the Warburg effect, is implicated in the carcinogenesis of $\mathrm{Ni}^{2}$ The elucidation of the impacts of $\mathrm{Ni}$ on cellular energy metabolism may be important for studying Ni toxicity. We found that miR-210 was an essential modulator of Ni-induced energy metabolism shift, resulting in oxygen consumption decrease, ATP deficit, alteration of HK activity, and accumulation of lactic acid. The key enzymes for OXPHOS, aconitase for the Krebs cycle and complex I for mitochondrial electron transfer, were suppressed by miR-210 without a protein level change of their component elements. It means that the inactivation of these enzymes accounts for the ISC deficit, rather than the inhibition of protein expression. Therefore, the repression of miR-210 on ISCU1/2 and downstream ISC-depended metabolic enzymes provides a logical explanation for the HIF-1 $\alpha$ stabilization and energy metabolism shift, both of which are observed under different Ni exposure conditions. ${ }^{11,29}$ Moreover, deficiencies in ISC-containing metabolic enzymes may attribute to the generation of reactive oxygen species (ROS) and mitochondria dysfunction, through which $\mathrm{Ni}$ induced significant cytotoxicity in nerve cells, as previously reported. ${ }^{27,37}$ In addition to $\mathrm{Ni}$, other heavy metals, including cobalt, manganese, and vanadium, induce HIF-1 $\alpha$ stabilization. ${ }^{28}$ Hence, the functional study of miR-210 may be valuable for the elucidation of heavy metal toxicity.

In contrast to low oxygen conditions, the hypoxia-mimic response induced by $\mathrm{Ni}$ may be accompanied by alterations of cellular iron levels. As previously reported, Ni may compete with iron for divalent metal transporter-1 (DMT1) and disturb cellular iron homeostasis. ${ }^{22,38}$ Because cellular iron levels are directly linked to the assembly of ISCs and ISC-dependent enzymes activity, ${ }^{39}$ the influence of $\mathrm{Ni}$ on cellular iron levels should not be excluded. However, based on our results that cellular exposure to $1 \mathrm{mM} \mathrm{NiCl}_{2}$ for $4 \mathrm{~h}$ did not disturb cellular iron homeostasis, we concluded that miR-210-mediated suppression of ISC-containing metabolic enzymes was independent from cellular iron regulation in the time frame of our Ni treatments. Costa and colleagues ${ }^{38}$ have reported the effects of $\mathrm{NiCl}_{2}$ on cellular iron regulation. In their study, a series of significant alterations that reflect the activation of cellular iron regulation emerged at $6-8 \mathrm{~h}$ post $\mathrm{Ni}$ exposure, which was consistent with the results of our study. Only the change in cellular iron content after $4 \mathrm{~h}$ of $\mathrm{Ni}$ exposure was different between the two studies that may be attributed to the use of different cell lines or methods of iron content determination. It appears that miR-210 modulated the energy metabolism shift before cellular iron homeostasis imbalance. Further studies are needed to identify the specific contributions of these mechanisms to the toxicity of $\mathrm{Ni}$. 

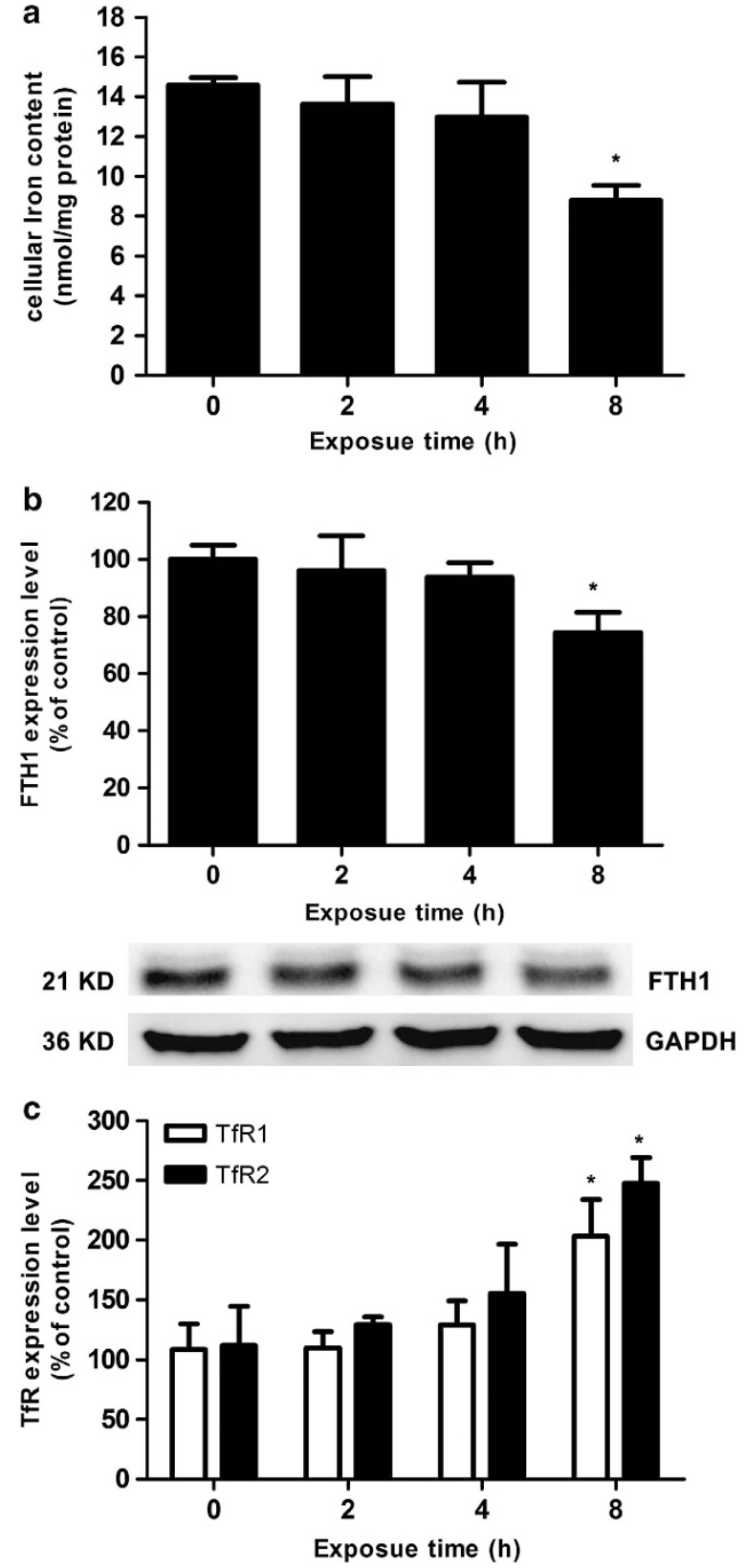

Figure 5 The nickel treatment containing $1 \mathrm{mM} \mathrm{NiCl}_{2}$ exposed to cells for $4 \mathrm{~h}$ does not disturb cellular iron homeostasis. (a) The cellular iron (ferrous and ferric) ions were measured with the Iron Assay Kit after exposure to $1 \mathrm{mM} \mathrm{NiCl} 2$ for different time periods $(0,2,4$, and $8 \mathrm{~h})$. A significant decrease in cellular iron ions was only found in the $8 \mathrm{~h}$ exposure groups. (b) The protein levels of total ferritin in cells that were treated with $1 \mathrm{mM} \mathrm{NiCl}_{2}$ for $0,2,4$, and $8 \mathrm{~h}$ were analyzed by western blot. Exposure to $1 \mathrm{mM} \mathrm{NiCl}_{2}$ for 0,2 , or $4 \mathrm{~h}$ does not alter the cellular ferritin level. (c) The transcript levels of the transferrin receptor (TfR), TfR1 and TfR2, were investigated by qRT-PCR after $1 \mathrm{mM} \mathrm{NiCl} l_{2}$ exposure in cells for $0,2,4$, and $8 \mathrm{~h}$. The overexpression of these two genes was only found in the $8 \mathrm{~h}$ exposure groups. The error bars reflect the S.E.M. of at least three independent experiments. ${ }^{*} P<0.05$ compared with the control

In summary, our findings suggest that the HIF- $1 \alpha$-controlling microRNA, miR-210, modulated the cellular energy metabolism shift by repressing ISCU1/2 after Ni exposure. Because energy metabolism is critical in the central nervous system, our results provide insight into the understanding of
Ni-mediated neurotoxicity. Furthermore, the ability of miR-210 to regulate hypoxia-mimic response by binding to target genes could be utilized to elucidate the health effects of a variety of heavy metals that induce HIF- $1 \alpha$ stabilization.

\section{Materials and Methods}

Cell culture and treatments. Mouse neuroblastoma cell lines (Neuro-2a), obtained from the Institute of Biochemistry and Cell Biology (Chinese Academy of Science, Shanghai, China), were cultured in Dulbecco's modified Eagle's medium (DMEM; Invitrogen, Carlsbad, CA, USA) supplemented with $10 \%$ heat-inactivated fetal bovine serum (FBS, HyClone, Logan, UT, USA) and $1 \% \mathrm{v} / \mathrm{v}$ penicillin/ streptomycin (Sigma-Aldrich, St. Louis, MO, USA) in a $5 \% \mathrm{CO}_{2}$-humidified atmosphere at $37^{\circ} \mathrm{C}$. A $1 \mathrm{M}$ stock solution of $\mathrm{Ni}$ chloride $\left(\mathrm{NiCl}_{2} \cdot 6 \mathrm{H}_{2} \mathrm{O}\right.$, SigmaAldrich) was prepared with sterile $\mathrm{H}_{2} \mathrm{O}$ and diluted to the appropriate concentrations for cell treatment.

Western blot analysis. From whole-cell extracts, $40 \mu \mathrm{g}$ of protein was resolved by SDS-PAGE and transferred onto a polyvinylidene fluoride membrane (Bio-Rad, Hercules, CA, USA). Membranes were probed for various proteins with the appropriate primary antibodies and visualized with the electrochemiluminescence system (Thermo Fisher Scientific, Waltham, MA, USA). The bands were imaged and analyzed using a ChemiDoc XRS + System with Image Lab Software (Bio-Rad).

Antibodies. Mouse monoclonal antibody to HIF- $1 \alpha$ was purchased from Novus Biologicals (Littleton, CO, USA). Rabbit monoclonal antibodies to HIF-1 $\beta$ and FTH1 were purchased from Cell Signaling Technology (Danvers, MA, USA). Rabbit polyclonal antibody to ISCU1/2 was purchased from Santa Cruz Biotechnology (Santa Cruz, CA, USA). Rabbit polyclonal antibody to Aconitase 2 and mouse monoclonal antibody to NDUFA9 were purchased from Abcam (Cambridge, UK)

Cell transfection. The miRNA mimic (miR10000267) or inhibitor (miR20000267) for cell transfection were purchased from RiboBio (Guangzhou, China) and used according to the manufacturer's instructions. Briefly, $24 \mathrm{~h}$ after cell seeding, the medium without penicillin/streptomycin was replaced with the transfection medium containing $100 \mathrm{nM}$ miRNA mimic or inhibitor and $0.2 \% \mathrm{v} / \mathrm{v}$ Lipofectamine 2000 transfection reagent (Invitrogen). After $4 \mathrm{~h}$, the transfection medium was replaced with fresh penicillin/streptomycin absent medium. The transiently transfected cells were collected after an additional 24-h incubation for further experiments.

qRT-PCR analysis. The extraction of total RNA and quantification of ISCU, TfR1, TfR2, and miR-210 were performed following our previously published protocol. ${ }^{27}$ The bulge-loop miRNA qRT-PCR primer sets (one reverse transcription primer and a pair of quantitative PCR primers for each set, MQP-0102 and MQP-0201) were designed by RiboBio. U6 was utilized as an internal control for miRNAs, and $\beta$-actin was used as an internal control for mRNA. Primers used in this study were as follows: ISCU-forward, $5^{\prime}$-CTCTGCCTGCCGCCTGTG- $3^{\prime}$ and reverse, 5'-CTGCTTCTCTGGCTCCTCCTTC-3'; TfR1-forward, 5'-GAGACTAC TTCCGTGCTACTTC-3' and reverse, $5^{\prime}$-TGGAGATACATAGGGCGACAG-3'; TfR2-forward, 5'-AGAGTGGTTGGAGGGCTAC-3' and reverse, 5'-CAATGAG GCTGACGAGAAGG- $3^{\prime} ; \beta$-actin-forward, $5^{\prime}$-CCACACCCGCCACCAGTTC- $3^{\prime}$ and reverse, $5^{\prime}$-CCTTCTGACCCATTCCCACCATC-3'.

Luciferase assay. The $3^{\prime}$-UTR regions of the ISCU containing the predicted binding sites for miR-210, wild or mutant (71-77 ACGCACA mutated to AGCGAGA), were cloned into pmiR-RB-REPORT vectors (GUR100509 and GUR100510; RiboBio). After co-transfection with the vectors and miR-210 mimic, the firefly and Renilla luciferase activities were measured using the Dual-luciferase Reporter Assay (Promega, Madison, WI, USA).

CCK assay. Cells were seeded into a 96-well plate and exposed to $1 \mathrm{mM} \mathrm{NiCl}_{2}$ for $2 \mathrm{~h}$. Next, 2-DG $(10 \mathrm{mM})$ or BrPA $(25 \mu \mathrm{M})$ was added to the Ni-containing medium and incubated for an additional $2 \mathrm{~h}$. Then, the medium in the 96 -well plate was replaced with fresh medium containing $10 \%$ v/v CCK-8 solution (Dojindo, Kumamoto, Japan) and incubated for $2 \mathrm{~h}$. The optical density (OD) of each well was determined at a wavelength of $450 \mathrm{~nm}$ with the Infinite M200 Microplate Reader (Tecan, Männedorf, Switzerland). Cell viability is reported as a percent of the control value. 
ATP assay. The ATP content was determined using the ATP Determination Kit (Molecular Probes, Eugene, OR, USA). The luminescence of the sample was measured using an Infinite M200 Microplate Reader (Tecan). ATP standard curves were established, and the ATP concentrations were expressed as $\mathrm{nmol} / \mathrm{mg}$ of protein.

Oxygen consumption rate. Cells $\left(1 \times 10^{5}\right.$ per well) were plated, transfected, and exposed to $\mathrm{NiCl}_{2}$ for $4 \mathrm{~h}$ before measurement. The rate of change of oxygen in media surrounding intact cells was measured by a XF96 Extracellular Flux Analyzer (Seahorse Bioscience, Billerica, MA, USA). The OCR of a well was determined by quantifying oxygen-dependent changes in fluorescence of a proprietary fluorescein complex (pmol/min/well).

HK activity assay. The activity of HK was measured using the Activity of Hexokinase Determination Kit (Nanjing Jiancheng, Nanjing, China) based on a coupled reaction of HK and 6-phosphoglucose dehydrogenase. The activity of hexokinase was determined by the generation of triphosphopyridine nucleotide (NADPH) and measured as the change in OD at $340 \mathrm{~nm}(\triangle \mathrm{OD} 340)$ at $37^{\circ} \mathrm{C}$ over $3 \mathrm{~min}$. The activity of hexokinase was expressed as $\Delta \mathrm{mOD} 340 / \mathrm{min} / \mathrm{mg}$ protein $(\Delta \mathrm{mOD} 340=\Delta \mathrm{OD} 340 / 1000)$.

Lactate assay. The intracellular lactate content was measured using the Lactate Determination Kit (Nanjing Jiancheng) according to the manufacturer's instructions. Standard curves were established, and the lactate concentrations were expressed as $\mu \mathrm{mol} / \mathrm{mg}$ of protein.

Aconitase and complex I activity assays. After appropriate treatment, cells were collected in Eppendorf tubes, resuspended in a hypotonic buffer $(120 \mathrm{mM} \mathrm{KCl}, 20 \mathrm{mM}$ HEPES, $2 \mathrm{mM} \mathrm{MgCl}$, and $1 \mathrm{mM}$ EGTA, pH 7.4) and disrupted by performing ten 1-s pulses with the Vibra-Cell ultrasonic processor (Sonics \& Materials, Danbury, CT, USA). The subsequent assessments of the two typical ISC metabolic enzymes were performed according to the previously reported protocol. ${ }^{12}$

Cellular iron content. The total cellular iron (ferrous and ferric) ions were measured using the Iron Assay Kit (Abcam) following the manufacturer's instructions. Cells were seeded into six-well plates and exposed to Ni for different time periods. Standard curves for the iron ions were established, and the cellular iron content was expressed as nmol per well.

Statistical analysis. Values are expressed as the mean \pm S.E.M. and were analyzed by SPSS (V18.0.0, SPSS Inc., Chicago, IL, USA). Data comparisons were completed using one-way ANOVA or paired $t$-test to compare the means of the different treatment groups. $P<0.05$ was considered statistically significant.

\section{Conflict of Interest}

The authors declare no conflict of interest.

Acknowledgements. We thank Dr. Chunhai Chen and Zhujun Zhang for their hospitable and professional technical assistance. Grant support was provided by the National Natural Science Foundation of China (No. 81102108) and the Outstanding Youth Science Fund of Chongqing (No. CSTC2013JCYJJQ10002).

1. Das KK, Das SN, Dhundasi SA. Nickel, its adverse health effects \& oxidative stress. Indian J Med Res 2008; 128: 412-425.

2. Costa M, Davidson TL, Chen $H, K e$, Zhang $P$, Yan $Y$ et al. Nickel carcinogenesis: epigenetics and hypoxia signaling. Mutat Res Fund Mol M 2005; 592: 79-88.

3. Kessler R. Nickel ENMs activate HIF-1alpha. Environ Health Perspect 2011; 119: A512.

4. Andrew AS, Klei LR, Barchowsky A. Nickel requires hypoxia-inducible factor-1 alpha, not redox signaling, to induce plasminogen activator inhibitor-1. Am J Physiol Lung Cell Mol Physiol 2001; 281: L607-L615.

5. Brant KA, Fabisiak JP. Nickel and the microbial toxin, MALP-2, stimulate proangiogenic mediators from human lung fibroblasts via a HIF-1alpha and COX-2-mediated pathway. Toxicol Sci 2009; 107: 227-237.

6. Viemann D, Schmidt M, Tenbrock K, Schmid S, Müller V, Klimmek K et al. The contact allergen nickel triggers a unique inflammatory and proangiogenic gene expression pattern via activation of NF-kappaB and hypoxia-inducible factor-1alpha. J Immunol 2007; 178: 3198-3207.
7. Feala JD, Coquin L, Zhou D, Haddad GG, Paternostro G, McCulloch AD. Metabolism as means for hypoxia adaptation: metabolic profiling and flux balance analysis. Bmc Syst Biol 2009; 3: 91.

8. Risbud MV, Guttapalli A, Stokes DG, Hawkins D, Danielson KG, Schaer TP et al. Nucleus pulposus cells express HIF-1 alpha under normoxic culture conditions: a metabolic adaptation to the intervertebral disc microenvironment. J Cell Biochem 2006; 98: 152-159.

9. Fijalkowska I, Xu W, Comhair SA, Janocha AJ, Mavrakis LA, Krishnamachary B et al. Hypoxia inducible-factor1alpha regulates the metabolic shift of pulmonary hypertensive endothelial cells. Am J Pathol 2010; 176: 1130-1138.

10. Frezza C, Zheng L, Tennant DA, Papkovsky DB, Hedley BA, Kalna G et al. Metabolic profiling of hypoxic cells revealed a catabolic signature required for cell survival. PLOS One 2011; 6: e24411.

11. Chen $\mathrm{H}$, Costa $\mathrm{M}$. Effect of soluble nickel on cellular energy metabolism in A549 cells. Exp Biol Med (Maywood) 2006; 231: 1474-1480.

12. He MD, Xu SC, Zhang $X$, Wang $Y$, Xiong JC, Zhang $X$ et al. Disturbance of aerobic metabolism accompanies neurobehavioral changes induced by nickel in mice. Neurotoxicology 2013; 38: 9-16.

13. Bhaskaran M, Mohan M. MicroRNAs: history, biogenesis, and their evolving role in animal development and disease. Vet Pathol 2013; e-pub ahead of print 17 September 2013; doi:10.1177/0300985813502820.

14. Kulshreshtha R, Ferracin M, Negrini M, Calin GA, Davuluri RV, Ivan M. Regulation of microRNA expression: the hypoxic component. Cell Cycle 2007; 6: 1426-1431.

15. Chan SY, Zhang YY, Hemann C, Mahoney CE, Zweier JL, Loscalzo J. MicroRNA-210 controls mitochondrial metabolism during hypoxia by repressing the iron-sulfur cluster assembly proteins ISCU1/2. Cell Metab 2009; 10: 273-284.

16. Lee DC, Romero R, Kim JS, Tarca AL, Montenegro D, Pineles BL et al. miR-210 targets iron-sulfur cluster scaffold homologue in human trophoblast cell lines: siderosis of interstitial trophoblasts as a novel pathology of preterm preeclampsia and small-forgestational-age pregnancies. Am J Pathol 2011; 179: 590-602.

17. Tong W-H, Rouault TA. Functions of mitochondrial ISCU and cytosolic ISCU in mammalian iron-sulfur cluster biogenesis and iron homeostasis. Cell Metab 2006; 3: 199-210.

18. Chen Z, Li Y, Zhang H, Huang P, Luthra R. Hypoxia-regulated microRNA-210 modulates mitochondrial function and decreases ISCU and COX10 expression. Oncogene 2010; 29: 4362-4368.

19. Favaro E, Ramachandran A, McCormick R, Gee H, Blancher C, Crosby M et al. MicroRNA-210 regulates mitochondrial free radical response to hypoxia and krebs cycle in cancer cells by targeting iron sulfur cluster protein ISCU. PLoS One 2010; 5: e10345.

20. Merlo A, de Quiros SB, Secades P, Zambrano I, Balbín M, Astudillo A et al. Identification of a signaling axis HIF-1alpha/microRNA-210/ISCU independent of SDH mutation that defines a subgroup of head and neck paragangliomas. J Clin Endocrinol Metab 2012; 97: E2194-E2200.

21. Muralimanoharan S, Maloyan A, Mele J, Guo C, Myatt LG, Myatt L. MIR-210 modulates mitochondrial respiration in placenta with preeclampsia. Placenta 2012; 33: 816-823.

22. Davidson T, Chen H, Garrick MD, D'Angelo G, Costa M. Soluble nickel interferes with cellular iron homeostasis. Mol Cell Biochem 2005; 279: 157-162.

23. Shiao YH, Lee SH, Kasprzak KS. Cell cycle arrest, apoptosis and p53 expression in nickel(II) acetate-treated Chinese hamster ovary cells. Carcinogenesis 1998; 19: 1203-1207.

24. Chakrabarti SK, Bai C. Role of oxidative stress in nickel chloride-induced cell injury in rat renal cortical slices. Biochem Pharmacol 1999; 58: 1501-1510.

25. Carroll S, Wood EJ. Exposure of human keratinocytes and fibroblasts in vitro to nickel sulphate ions induces synthesis of stress proteins Hsp72 and Hsp90. Acta Derm Venereol 2000; 80: 94-97.

26. Trombetta D, Mondello MR, Cimino F, Cristani M, Pergolizzi S, Saija A. Toxic effect of nickel in an in vitro model of human oral epithelium. Toxicol Lett 2005; 159: 219-225.

27. He MD, Xu SC, Lu YH, Li L, Zhong M, Zhang YW et al. L-carnitine protects against nickel-induced neurotoxicity by maintaining mitochondrial function in Neuro-2a cells. Toxicol Appl Pharmacol 2011; 253: 38-44.

28. Kang GS, Li Q, Chen H, Costa M. Effect of metal ions on HIF-1alpha and Fe homeostasis in human A549 cells. Mutat Res 2006; 610: 48-55.

29. Pietruska JR, Liu X, Smith A, McNeil K, Weston P, Zhitkovich A et al. Bioavailability, intracellular mobilization of nickel, and HIF-1alpha activation in human lung epithelial cells exposed to metallic nickel and nickel oxide nanoparticles. Toxicol Sci 2011; 124: 138-148.

30. Huang X, Le QT, Giaccia AJ. MiR-210-micromanager of the hypoxia pathway. Trends Mol Med 2010; 16: 230-237.

31. Fasanaro P, D'Alessandra Y, Di Stefano V, Melchionna R, Romani S, Pompilio G et al. MicroRNA-210 modulates endothelial cell response to hypoxia and inhibits the receptor tyrosine kinase ligand Ephrin-A3. J Biol Chem 2008; 283: 15878-15883.

32. Hu SJ, Huang M, Li Z, Jia F, Ghosh Z, Lijkwan MA et al. MicroRNA-210 as a novel therapy for treatment of ischemic heart disease. Circulation 2010; 122: S124-S131.

33. Giannakakis A, Sandaltzopoulos R, Greshock J, Liang S, Huang J, Hasegawa K et al. miR-210 links hypoxia with cell cycle regulation and is deleted in human epithelial ovarian cancer. Cancer Biol Ther 2008; 7: 255-264.

34. Zhang Z, Sun H, Dai H, Walsh RM, Imakura M, Schelter J et al. MicroRNA miR-210 modulates cellular response to hypoxia through the MYC antagonist MNT. Cell Cycle 2009; 8: $2756-2768$. 
35. Chio CC, Lin JW, Cheng HA, Chiu WT, Wang YH, Wang JJ et al. MicroRNA-210 targets antiapoptotic Bcl-2 expression and mediates hypoxia-induced apoptosis of neuroblastoma cells. Arch Toxicol 2013; 87: 459-468.

36. Everts B, Freitas TC, Chott R, Yarasheski KE, Pearce EL, Pearce EJ et al. Commitment to glycolysis sustains survival of NO-producing inflammatory dendritic cells. Blood 2012; 120 1422-1431.

37. Xu SC, He MD, Zhong M, Zhang YW, Wang Y, Yang L et al. Melatonin protects against Nickel-induced neurotoxicity in vitro by reducing oxidative stress and maintaining mitochondrial function. J Pineal Res 2010; 49: 86-94.

38. Chen H, Davidson T, Singleton S, Garrick MD, Costa M. Nickel decreases cellular iron level and converts cytosolic aconitase to iron-regulatory protein 1 in A549 cells. Toxicol Appl Pharmacol 2005; 206: 275-287.
39. Lill R, Hoffmann B, Molik S, Pierik AJ, Rietzschel N, Stehling 0 et al. The role of mitochondria in cellular iron-sulfur protein biogenesis and iron metabolism. Biochim Biophys Acta 2012; 1823: 1491-1508.

(c) (-) Cell Death and Disease is an open-access journal published by Nature Publishing Group. This work is licensed under a Creative Commons Attribution-NonCommercialShareAlike 3.0 Unported License. To view a copy of this license, visit http://creativecommons.org/licenses/by-nc-sa/3.0/

Supplementary Information accompanies this paper on Cell Death and Disease website (http://www.nature.com/cddis) 